\title{
Human blood proteins and correlations with biochemical parameters after long duration space flights
}

\author{
D.N. Kashirina ${ }^{1 *}$, L.Kh. Pastushkova ${ }^{1}$, A.M. Nosovsky ${ }^{1}$, A.J. Percy ${ }^{2}$, \\ K.S. Kireev ${ }^{3}$, C.H. Borchers ${ }^{2}$, E.N. Nikolaev ${ }^{4}$, I.M. Larina ${ }^{1}$ \\ ${ }^{1}$ Institute for Biomedical Problems - Russian Federation State Scientific Research Center RAS, \\ Moscow, Russia \\ ${ }^{2}$ Genome British Columbia Proteomics Centre, University of Victoria, Victoria, BC, Canada \\ ${ }^{3}$ Yu.A. Gagarin Research and Test Cosmonaut Training Center, Star City, Moscow Region, Russia \\ ${ }^{4}$ Skolkovo Institute of Science and Technology, Skoltech, Moscow region, Russia \\ *e-mail: daryakudryavtseva@mail.ru
}

Key words: blood plasma, cosmonauts, mass spectrometry, biochemical parameters

Motivation and Aim: The peculiar features of space radiation and microgravity pose a risk to the cardiovascular system (CVS) and add to the risks of manned space exploration. Accelerated 'ageing-like' changes develop in the CVS during spaceflight. Over a 6-month stay on the ISS, vascular and cardiac structural and functional modifications occur, including increased vascular wall thickness and stiffness, which might predispose astronauts to atherogenesis [1]. However the molecular mechanisms driving physiological changes remain unknown.

Methods and Algorithms: Blood samples from 18 Russian cosmonauts who had conducted long-duration space flights were analyzed. The samples were collected 30 days before launch, on the first and seventh days after landing. Biochemical parameters of blood were measured at the same time points. Using mass spectrometry approach involving multiple reaction monitoring in conjunction with stable isotope-labeled standards at the University of Victoria, 125 plasma proteins were detected and quantitated. To determine the dependencies between proteins and biochemical parameters of blood, a correlation was made. To increase the accuracy of the analysis, the group of cosmonauts was divided into 2 homogeneous groups, correlations were determined in each group.

Results: It was revealed that in both groups there was a correlation between total cholesterol and apolipoprotein B-100, LDL and cystacin-C and apolipoprotein E, triglycerides and coagulation factor VII and apolipoprotein C-II. These proteins could be a potential risk factors for cardiovascular disease. It was found that paraoxonase-1 correlated positively with HDL and apolipoprotein A-I00, and negatively with the coefficient of atherogenicity. Therefore this protein could have a protective effect on blood vessels.

Conclusion: The correlations found are partially confirmed by other authors and methods, and in part - it allow to construct hypotheses about the unexplored mechanisms of cardiovascular dysfunction after space flight.

Acknowledgements: The work was supported by RFBR grant No. 18-34-00524. We express special thanks to Bruker Daltonics for financial and technical support.

\section{References}

1. Hughson R.L., Helm A., Durante M. (2018) Heart in space: effect of the extraterrestrial environment on the cardiovascular system. Nat Rev Cardiol. 15(3):167-180. DOI 10.1038/nrcardio.2017.157. 